\title{
Algebraic Analysis of Minimal Representations
}

\author{
Dedicated to Mikio Sato whose pioneering work \\ in algebraic analysis has been an inspiration for me
}

by

Toshiyuki KoBAYASHI

\begin{abstract}
Small representations of a group yield large symmetries in the representation space. Analysis of minimal representations utilizes large symmetries in their geometric models, and serves as a driving force in creating new interesting problems that interact with other branches of mathematics.

This article discusses the following three topics that arise from minimal representations of the indefinite orthogonal group:

1. construction of conservative quantities for ultra-hyperbolic equations,

2. quantitative discrete branching laws,

3. deformation of the Fourier transform,

with emphasis on the prominent role of Sato's ideas in algebraic analysis.

2010 Mathematics Subject Classification: Primary 22E46; Secondary 22E45, 53A30, 46F15, $58 \mathrm{~J} 15$.

Keywords: minimal representations, hyperfunction, branching law, reductive group, generalized Fourier transform, holomorphic semigroup, conservative quantity, $\mathcal{D}$-module.
\end{abstract}

\section{$\S 1$. Introduction}

The aim of this article is to highlight the prominent role of Sato's idea of hyperfunctions and $\mathcal{D}$-modules in the new developments in the analysis of minimal representations [4, 35, 36, 40.

Minimal representations are the simplest, infinite-dimensional 'unipotent representations'. They are building blocks of unitary representations. The Segal-

This is a contribution to the special issue "The golden jubilee of algebraic analysis".

Communicated by M. Kashiwara. Received December 31, 2009.

T. Kobayashi: Graduate School of Mathematical Sciences and IPMU, The University of Tokyo, 3-8-1 Komaba, Meguro, Tokyo, 153-8914 Japan;

e-mail: toshi@ms.u-tokyo.ac.jp

(C) 2011 Research Institute for Mathematical Sciences, Kyoto University. All rights reserved. 
Shale-Weil representation is a classic example for the split simple group of type C. There has been an active study of minimal representations of reductive groups, mostly through algebraic approaches since 1990s both over the real and $p$-adic fields [5, 6, 10, 12, 22, 23, 37, 42, 43, 46, 50, 52.

On the other hand, I believe that geometric analysis of minimal representations is also a promising area, and I have been advocating its study based on the following change of viewpoint:

$$
\begin{aligned}
& \text { small representations of a group } \\
= & \text { large symmetries in a representation space. }
\end{aligned}
$$

The terminology 'minimal representations' is defined inside representation theory (i.e. the annihilator in the universal enveloping algebra is the Joseph ideal, see e.g. 10 ), and the corresponding 'largest symmetries' are expected to serve as a driving force in creating new interesting areas of mathematics even outside representation theory.

The 'largest symmetries' in representation spaces of minimal representations may also be observed in branching laws. Indeed, as we shall see in Theorem 3.4 . it may well happen that broken symmetries of minimal representations reduce to analysis of certain semisimple symmetric spaces (see also [30, 39, 41]). This observation indicates that analysis of minimal representations involves higher symmetries than those for (traditional) analysis of symmetric spaces.

We focus on the minimal representation of a simple group of type D. This is just a single irreducible representation, but it turns out that geometric analysis of its various models is surprisingly rich. Indeed, papers devoted to this single representation in very recent years already exceed 500 pages, giving rise to interactions with the following topics:

- conformal geometry for general pseudo-Riemannian manifolds [30, 38,

- Dolbeault cohomologies on open complex manifolds 37,

- conservative quantities for ultra-hyperbolic equations [40,

- breaking symmetries and discrete branching laws 39],

- Schrödinger model and the unitary inversion operator [34, 36,

- deformation of Fourier transforms [4,

- holomorphic semigroups [33, 35],

- new special function theory for fourth order differential operators [16, 17].

In this article, we choose three topics among them, and try to explain their flavour in Sections 2, 3 and 4 respectively, with emphasis on the role of Sato's 
ideas in algebraic analysis, both in philosophy and in techniques. For the reader's convenience, we list some representation-theoretic properties of our minimal representation in the Appendix.

\section{$\S 2$. Conservative quantities for $\mathcal{D}$-modules}

The energy of a wave is a conservative quantity for the wave equation, namely, it is invariant under time-translations. In this section, we discuss higher symmetries coming from conformal transformations. By using the idea of Sato's hyperfunctions [20, 47, we construct conservative quantities for specific ultra-hyperbolic equations (see Theorem 2.6).

\section{§2.1. Yamabe operator and conformal geometry}

A diffeomorphism $h$ of a Riemannian manifold $(X, g)$ is said to be conformal if there exists a positive-valued function $\Omega(h, \cdot)$ on $X$ such that

$$
h^{*} g_{h x}=\Omega(h, x)^{2} g_{x} \quad \text { for } x \in X .
$$

It is an isometry if $\Omega(h, \cdot) \equiv 1$. We write

$$
\operatorname{Isom}(X, g) \subset \operatorname{Conf}(X, g)
$$

for the groups consisting of the isometries and conformal diffeomorphisms, respectively. The same notation will be applied to a more general setting where $g$ is a non-degenerate symmetric tensor, namely, to an indefinite-Riemannian manifold.

The invariance for the Laplacian $\Delta_{X}$ characterizes isometries among diffeomorphisms of $X$. In other words, a non-isometric transformation on $(X, g)$ does not preserve $\Delta_{X}$. However, the Laplacian $\Delta_{X}$ is still subject to the following covariance under conformal transformations:

$$
\varpi_{(n+2) / 2}(h) \circ \widetilde{\Delta}_{X}=\widetilde{\Delta}_{X} \circ \varpi_{(n-2) / 2}(h) \quad \text { for any } h \in \operatorname{Conf}(X, g),
$$

where $n$ is the dimension of $X, \mathrm{Scal}_{X}$ is the scalar curvature, and

$$
\begin{aligned}
\widetilde{\Delta}_{X} & :=\Delta_{X}-\frac{n-2}{4(n-1)} \text { Scal }_{X} & & \text { (the Yamabe operator), } \\
\varpi_{\lambda}(h) f(x) & :=\Omega\left(h^{-1}, x\right)^{\lambda} f\left(h^{-1} x\right) & & \text { for } f \in C^{\infty}(X) .
\end{aligned}
$$

The formula 2.1) implies that the operator $\Delta_{X}$ (or $\widetilde{\Delta}_{X}$ ) is not conformally invariant, but the $\mathcal{D}$-module generated by $\widetilde{\Delta}_{X}$ is conformally invariant! As far as the solutions are concerned, the invariance of the $\mathcal{D}$-module is sufficient. Namely, 
by putting

$$
\operatorname{Sol}\left(\widetilde{\Delta}_{X}\right):=\left\{f \in C^{\infty}(X): \Delta_{X} f=\frac{n-2}{4(n-1)} \operatorname{Scal}_{X} f\right\}
$$

we get:

Fact 2.1. The conformal group $\operatorname{Conf}(X, g)$ preserves $\mathcal{S}$ ol $\left(\widetilde{\Delta}_{X}\right)$ via $\varpi_{(n-2) / 2}$.

See [38, Theorem 2.5] for the proof.

\section{Remark 2.2.}

(1) The eigenspaces $\mathcal{S}$ ol $\left(\widetilde{\Delta}_{X}-\lambda\right)$ are not conformally invariant if $\lambda \neq 0$.

(2) Fact 2.1 may be better formulated if we use the ring of twisted differential operators acting on sections of a certain line bundle.

(3) The differential equation $\widetilde{\Delta}_{X} f=0$, that is, $\Delta_{X} f=\frac{n-2}{4(n-1)} \operatorname{Scal}_{X} f$, is elliptic, hyperbolic, or ultra-hyperbolic if $(X, g)$ is Riemannian, Lorentzian, or of general signature, respectively.

Then a general problem is:

Problem 2.3 (see [30, Problem C]).

(1) Does there exist an invariant inner product on an appropriate subspace of $\operatorname{Sol}\left(\widetilde{\Delta}_{X}\right)$ ?

(2) If yes, construct it explicitly.

Such an inner product may be seen as a conservative quantity for the solution to the equation $\widetilde{\Delta}_{X} f=0$. Problem 2.3 does not find a final answer in the general setting. We shall give a partial answer in the flat case (see Theorem 2.6 below).

\section{$\S 2.2$. Conservative quantities}

Let $\mathbb{R}^{p, q}$ be the Euclidean space $\mathbb{R}^{p+q}$ endowed with the flat indefinite-Riemannian structure

$$
d s^{2}=d x_{1}^{2}+\cdots+d x_{p}^{2}-d x_{p+1}^{2}-\cdots-d x_{p+q}^{2} .
$$

Then the corresponding Laplace-Beltrami operator takes the form

$$
\square_{p, q}:=\frac{\partial^{2}}{\partial x_{1}^{2}}+\cdots+\frac{\partial^{2}}{\partial x_{p}^{2}}-\frac{\partial^{2}}{\partial x_{p+1}^{2}}-\cdots-\frac{\partial^{2}}{\partial x_{p+q}^{2}} .
$$

Obviously, the scalar curvature on $\mathbb{R}^{p, q}$ vanishes identically. Hence, the Yamabe operator on $\mathbb{R}^{p, q}$ coincides with $\square_{p, q}$. The space of solutions to $\square_{p, q} f=0$, denoted by $\operatorname{Sol}\left(\square_{p, q}\right)$, is obviously invariant under the motion group

$$
\operatorname{Isom}\left(\mathbb{R}^{p, q}\right) \simeq O(p, q) \ltimes \mathbb{R}^{p+q} .
$$


It was proved in [40, Theorem 4.7] that $\mathcal{S}$ ol $\left(\square_{p, q}\right)$ has even larger symmetries if $p+q$ is even, namely, under the indefinite orthogonal group

$$
G:=O(p+1, q+1)=\left\{g \in G L(p+q+2, \mathbb{R}):{ }^{t} g I_{p+1, q+1} g=I_{p+1, q+1}\right\}
$$

acting on $\mathbb{R}^{p+q}$ as Möbius transforms. (To be more precise, $G$ preserves the space $\operatorname{Sol}_{0}\left(\square_{p, q}\right)$ of smooth solutions with certain decay conditions at infinity together with their derivatives.)

\section{Remark 2.4.}

(1) The parity condition on $p+q$ is crucial. In fact, a theorem of Howe and Vogan 51] asserts that there does not exist an infinite-dimensional representation of $G$ whose Gelfand-Kirillov dimension is $p+q-1$ if $p+q$ is odd and $p, q>3$.

(2) $\operatorname{Sol}_{0}\left(\square_{p, q}\right)$ is defined as the twisted pull-back of smooth functions on the conformal compactification of $\mathbb{R}^{p+q}$. See [40] for details.

Problem 2.3 in this specific setting is stated as:

Problem 2.5. Find a G-invariant inner product on $\mathcal{S}_{0} l_{0}\left(\square_{p, q}\right)$ if it exists.

\section{§2.3. Unitarizability versus unitarization}

If $p, q>0$ and $p+q$ is even and greater than two, then we can tell a priori that the representation on $\mathcal{S o l}_{0}\left(\square_{p, q}\right)$ is unitarizable and irreducible (e.g. [5, 38]) by algebraic techniques. Namely, we know the existence and uniqueness of a $G$ invariant inner product on $\mathcal{S}_{0} l_{0}\left(\square_{p, q}\right)$ in this case.

What we seek in Problem 2.5 is not merely an abstract unitarizability but the unitarization of the representation space for a concrete geometric model, namely, the construction of an invariant inner product. There are two approaches to the unitarization: one is easier, and the other is more challenging as discussed below.

The easier approach to Problem 2.5 is to write the inner product by using the integral representation of solutions. Such an integral formula was given in [40, Theorem 4.7] by using an explicit formula for the Green kernel [30, 40]. The disadvantage of this approach is that the formula for the inner product involves a preimage of the integral representation, which is not canonically given.

A second approach is to use an expansion of solutions into a countable sum of better understood solutions, and then to give a Parseval-Plancherel type theorem. We shall discuss this approach in Section 3 .

A more intrinsic approach is to find a formula for the inner product directly without using the integral representation of solutions. A hint to this is the wellknown energy formula for the wave equation, which is given by integration of the 
Cauchy data on the hyperplane $(t=$ constant) in the space-time, (see $(2.6)$ ). (We note, however, that the energy is not conformally invariant but invariant only under time-translations.)

In order to explain the second approach, let us set up some notation. We recall that any non-characteristic hyperplane in $\mathbb{R}^{p, q}$ is written as

$$
\alpha \equiv \alpha_{v, c}:=\left\{x \in \mathbb{R}^{p+q}:(x, v)_{\mathbb{R}^{p, q}}=c\right\}
$$

for some $c \in \mathbb{R}$ and $v \in \mathbb{R}^{p, q}$ such that $(v, v)_{\mathbb{R}^{p, q}}= \pm 1$. Fix such a $v$, and express a function $f$ on $\mathbb{R}^{p+q}$ as Sato's hyperfunction (47]) in the direction of $v$, namely,

$$
f(x)=\lim _{\varepsilon \downarrow 0}\left(f_{+}(x+\sqrt{-1} \varepsilon v)-f_{-}(x-\sqrt{-1} \varepsilon v)\right) .
$$

Here, $f_{ \pm}(x+t v)$ is a holomorphic function of one variable $t$ near the real axis in $\pm \operatorname{Im} t>0$.

We set

$$
\frac{\partial f_{ \pm}}{\partial \nu}(x):=\left.\frac{\partial}{\partial t}\right|_{t=0} f(x+t v) \quad \text { (normal derivative) }
$$

and introduce a function $Q_{\alpha} f$ on the hyperplane $\alpha \equiv \alpha_{v, c}$ by

$$
Q_{\alpha} f:=\frac{1}{\sqrt{-1}}\left(f_{+} \frac{\overline{\partial f_{+}}}{\partial \nu}-f_{-} \frac{\overline{\partial f_{-}}}{\partial \nu}\right) .
$$

Finally, we define

$$
(f, f):=\int_{\alpha} Q_{\alpha} f
$$

The right-hand side of 2.5 does not always converge, but it makes sense if $f$ satisfies suitable decay conditions, say, $f \in \mathcal{S}_{0} l_{0}\left(\square_{p, q}\right)$. Then, we can give an answer to Problem 2.5 as follows:

Theorem 2.6 (see [40, Theorem 6.2], also [30]).

(1) For $f \in \mathcal{S}_{0} l_{0}\left(\square_{p, q}\right)$, 2.5 is independent of the choice of the pair $\left(f_{+}, f_{-}\right)$in the expression (2.4) and of the hyperplane $\alpha$.

(2) $(f, f) \geq 0$ for any $f \in \mathcal{S o l}_{0}\left(\square_{p, q}\right)$. Equality holds if and only if $f=0$.

(3) The polarization of the norm 2.5 yields a G-invariant inner product on $\operatorname{Sol}_{0}\left(\square_{p, q}\right)$.

We denote by $\overline{\mathcal{S} o l_{0}\left(\square_{p, q}\right)}$ the Hilbert space obtained as the completion of $\operatorname{Sol}_{0}\left(\square_{p, q}\right)$. Thus, we get a unitary representation of $G=O(p+1, q+1)$, to be denoted by $\varpi \equiv \varpi^{p+1, q+1}$, on $\overline{\mathcal{S}_{o l}\left(\square_{p, q}\right)}$. It turns out that this is an irreducible and 
minimal representation of $G$. See Section 5 for representation-theoretic properties of $\varpi$.

Remark 2.7. Assertion (1) in Theorem 2.6 is a part of the invariance of the inner product ( , ) because any non-characteristic hyperplane is conjugate to either $x_{1}=0$ or $x_{p+q}=0$ under the motion group $\operatorname{Isom}\left(\mathbb{R}^{p, q}\right) \simeq O(p, q) \ltimes \mathbb{R}^{p+q}$. We note that $G$ contains $\operatorname{Isom}\left(\mathbb{R}^{p, q}\right)$ as a proper subgroup.

The proof of Theorem 2.6 was given in [40] by using some representationtheoretic results on the representation $\varpi$. It might be interesting to find a proof that does not depend on group theory but only on geometry such as Stokes' theorem. We write this down as an open problem:

Problem 2.8. Give a purely geometric proof of Theorem 2.6 .

\section{§2.4. Energy generator}

Our conformally invariant inner product $(2.5)$ is very close to the energy of the wave, where one integrates Cauchy data on the zero time hyperplane. We end this section by making this connection more explicit.

For $p=1$, let us introduce time and space coordinates $(t ; x)$ instead of the previous coordinates $\left(x_{1}, \ldots, x_{p} ; x_{p+1}, \ldots, x_{p+q}\right)$. Then the energy of the wave $f$ is given by

$$
\mathcal{E}(f)=\frac{1}{2} \int_{\mathbb{R}^{q}}\left(\left|f_{t}\right|^{2}+|\nabla f|^{2}\right) d x .
$$

In terms of the inner product 2.5$), \mathcal{E}(f)$ is written as

$$
(f,|H| f)=\left(f^{+}, H f^{+}\right)-\left(f^{-}, H f^{-}\right)
$$

where $H=i \partial_{t}$ is the energy generator (infinitesimal time-translation). Since the energy generator $H$ is invariant under time-translations (i.e. invariant under a one-dimensional subgroup of $G$ ) and the inner product $($,$) is invariant under the$ whole group $G, \mathcal{E}(f)$ is also invariant under time-translations. This explains the classical fact that the energy (2.6) is a conservative quantity in the narrow sense that it is independent of which constant-time hyperplane we integrate over.

\section{§3. Quantitative branching laws}

In Section 1. we have given a concrete formula for the conformally invariant inner product on the minimal representation (conservative quantities). It is given by an integral over hyperplanes. Yet another formula for the same inner product will be given as a countable sum of well-understood quantities. 
This is a Parseval-type theorem (see Theorem 3.4), which is built on a 'good expansion theorem' for solutions. Such an expansion theorem is obtained as a special case of the general theory of discretely decomposable restrictions of unitary representations (see Theorem 3.2. We will see that algebraic analysis provides a powerful method for solving branching problems in representation theory (cf. Problem 3.1 below).

\section{$\S 3.1$. Breaking symmetries and discrete decomposability}

Suppose $\pi: G \rightarrow G L(\mathcal{H})$ is a unitary representation of a Lie group $G$. Given a subgroup $G^{\prime}$ of $G$, consider the broken symmetry, that is, the restriction $\left.\pi\right|_{G^{\prime}}$. In general, $\left.\pi\right|_{G^{\prime}}$ decomposes into a direct integral of irreducible representations of $G^{\prime}$. Our concern here is:

Problem 3.1 (see [24, 25]). For which triple $\left(G, G^{\prime}, \pi\right)$ does the restriction $\left.\pi\right|_{G^{\prime}}$ decompose discretely with finite multiplicities?

It often happens that the irreducible decomposition of $\left.\pi\right|_{G^{\prime}}$ (branching law) contains a continuous spectrum if $G^{\prime}$ is non-compact. Even worse, each irreducible representation of $G^{\prime}$ may occur in the branching law with infinite multiplicities. Thus, Problem 3.1 seeks for a very nice class of branching laws.

Now, let us fix some notation for a real reductive group $G$. Let $K$ be a maximal compact subgroup of $G, T$ a maximal torus of $K$, and $\mathfrak{t}, \mathfrak{k}$ the Lie algebras of $T, K$, respectively. We choose the set $\Delta^{+}(\mathfrak{k}, \mathfrak{t})$ of positive roots, and denote the dominant Weyl chamber by $\mathfrak{t}_{+}\left(\subset \sqrt{-1} \mathfrak{t}^{*}\right)$. We also fix a $K$-invariant inner product on $\mathfrak{k}$, and regard $\sqrt{-1} \mathfrak{t}^{*}$ as a subset of $\sqrt{-1} \mathfrak{k}^{*}$.

Suppose that $K^{\prime}$ is a closed subgroup of $K$. The group $K$ acts on the homogeneous space $K / K^{\prime}$ from the left, and then on the cotangent bundle $T^{*}\left(K / K^{\prime}\right)$ in a Hamiltonian fashion. We write

$$
\mu: T^{*}\left(K / K^{\prime}\right) \rightarrow \sqrt{-1} \mathfrak{k}^{*}
$$

for the momentum map, and define the closed cone

$$
C_{K}\left(K^{\prime}\right):=\text { Image } \mu \cap \mathfrak{t}_{+} \text {. }
$$

For a closed subgroup $G^{\prime}$ of $G$, we shall consider $C_{K}\left(K^{\prime}\right)$ with $K^{\prime}:=K \cap G^{\prime}$.

Next, suppose that $\pi$ is a (reducible) representation of a compact Lie group $K$. The asymptotic $K$-support of $\pi$, to be denoted by $\operatorname{AS}_{K}(\pi)$, was introduced by Kashiwara and Vergne [21] as the asymptotic cone of the $K$-types of $\pi$. By definition $\operatorname{AS}_{K}(\pi)=\{0\}$ if $\operatorname{dim} \pi<\infty$. For a representation $\pi$ of $G$, we can define $\operatorname{AS}_{K}(\pi)$ by considering the restriction $\left.\pi\right|_{K}$.

We are ready to state an answer to Problem 3.1 
Theorem 3.2 (see [26]). Suppose that $\pi$ is a unitary representation of $G$ of finite length, and that $G^{\prime}$ is a closed subgroup of $G$. Set $K^{\prime}=K \cap G^{\prime}$. If

$$
C_{K}\left(K^{\prime}\right) \cap \operatorname{AS}_{K}(\pi)=\{0\},
$$

then the restriction $\left.\pi\right|_{G^{\prime}}$ decomposes discretely into a direct sum of irreducible unitary representations of $G^{\prime}$ with finite multiplicities.

An upper estimate of the singularity spectrum of the hyperfunction character of $\pi$ plays a crucial role in the proof of Theorem 3.2. In particular, the assumption (3.1) ensures that

$$
\text { Restriction and Trace (hyperfunction character) commute. }
$$

Here, we remark that the character of an infinite-dimensional representation $\pi$,

$$
\operatorname{Trace} \pi(g) \quad(g \in G),
$$

does not make sense as an ordinary function because Trace $\pi(e)=\operatorname{dim} \pi=\infty$. Harish-Chandra proved that Trace $\pi$ is well-defined as a distribution on $G$ if $\pi$ is an irreducible unitary representation of a real reductive group $G$, and proved further that Trace $\pi$ belongs to $L_{\text {loc }}^{1}(G)$. On the other hand, the restriction Trace $\left.\pi\right|_{K}$ is not locally integrable on $K$ any more (see Atiyah [1]). What $(3.2$ means is that

$$
\operatorname{Trace}\left(\left.\pi\right|_{K^{\prime}}\right)=\left.\operatorname{Trace}(\pi)\right|_{K^{\prime}}
$$

as an identity of hyperfunctions (or distributions) on $K^{\prime}$. See [26, Theorem 2.8] for the proof. We also refer to the lecture notes 32 for heuristic ideas of the proof.

Recently, Hansen, Hilgert, and Keliny [13] have given an alternative proof of Theorem 3.2 by replacing Sato's hyperfunctions with Schwartz's distributions. See also [27, 28] for a necessary condition of discrete decomposability of branching laws, where the associated variety of an infinite-dimensional representation $\pi$ (an analogue of the characteristic variety of a $\mathcal{D}$-module) plays an important role. The references [29, 31] discuss some applications of discrete branching laws.

Loosely, Theorem 3.2 says that if $C_{K}\left(K^{\prime}\right)$ and $\mathrm{AS}_{K}(\pi)$ are not 'large' then the restriction $\left.\pi\right|_{G^{\prime}}$ is discretely decomposable. We note that $C_{K}\left(K^{\prime}\right)=\{0\}$ if $K^{\prime}=K$, and consequently the assumption (3.1) is automatically satisfied. In this case, Theorem 3.2 is precisely Harish-Chandra's admissibility theorem ([14]). For any minimal representation $\pi$ of a reductive group $G$, we know from [51] that $\operatorname{AS}_{K}(\pi)$ is one-dimensional, i.e. $\operatorname{AS}_{K}(\pi)=\mathbb{R} v$ or $\mathbb{R}_{+} v$ where $v$ is the highest root. Thus we can expect that there is a rich family of subgroups $G^{\prime}$ of $G$ for which the restriction of the minimal representation of $G$ decomposes discretely. 


\section{§3.2. Space forms of indefinite-Riemannian manifolds}

Before applying Theorem 3.2 to actual branching problems, we review quickly known results about the geometry and global analysis on space forms of indefiniteRiemannian manifolds (also referred to as pseudo-hyperbolic spaces, generalized hyperboloids, etc.).

We set

$$
\begin{aligned}
& X_{+}^{p, q}:=\left\{(x, y) \in \mathbb{R}^{p+1} \oplus \mathbb{R}^{q}:\|x\|^{2}-\|y\|^{2}=1\right\} \simeq O(p+1, q) / O(p, q), \\
& X_{-}^{p, q}:=\left\{(x, y) \in \mathbb{R}^{p} \oplus \mathbb{R}^{q+1}:\|x\|^{2}-\|y\|^{2}=-1\right\} \simeq O(p, q+1) / O(p, q) .
\end{aligned}
$$

We note that $X_{+}^{p, 0} \simeq S^{p}$ and $X_{-}^{0, q} \simeq S^{q}$. By switching the factors, we have $X_{+}^{p, q}$ $\simeq X_{-}^{q, p}$.

We induce an indefinite-Riemannian structure on $X_{+}^{p, q}$ and $X_{-}^{p, q}$ from the ambient space $\mathbb{R}^{p+1, q}$ and $\mathbb{R}^{p, q+1}$, respectively. Then, $X_{+}^{p, q}$ and $X_{-}^{p, q}$ have constant sectional curvatures. Here is a summary of the properties of $X_{+}^{p, q}$ and $X_{-}^{p, q}$ :

\begin{tabular}{lcc}
\hline & Sectional curvature $\kappa$ & Signature of metric tensor \\
\hline$X_{+}^{p, q}$ & $\kappa \equiv+1$ & $(p, q)$ \\
$X_{-}^{p, q}$ & $\kappa \equiv-1$ & $(p, q)$ \\
\hline
\end{tabular}

Let $L^{2}\left(X_{+}^{p-1, q}\right)$ be the Hilbert space of square integrable functions on $X_{+}^{p-1, q}$ with respect to the induced volume element. For $\lambda \in \mathbb{C}$, we set

$$
V_{\lambda}^{p, q}:=\left\{f \in L^{2}\left(X_{+}^{p-1, q}\right): \widetilde{\Delta}_{X_{+}^{p-1, q}} f=\left(1 / 4-\lambda^{2}\right) f\right\},
$$

where the Yamabe operator $\widetilde{\Delta}_{X_{+}^{p, q}}$ takes the following form:

$$
\widetilde{\Delta}_{X_{+}^{p, q}}=\Delta_{X_{+}^{p, q}}-\frac{1}{4}(p+q)(p+q-2) .
$$

Clearly, the isometry group $\operatorname{Isom}\left(X_{+}^{p-1, q}\right) \simeq O(p, q)$ preserves $V_{\lambda}^{p, q}$ for any $\lambda \in \mathbb{C}$. The representations on $V_{\lambda}^{p, q}$ are called discrete series representations for $X_{+}^{p-1, q}$ if $V_{\lambda}^{p, q} \neq\{0\}$, which were studied by Gelfand, Graev, Vilenkin, Shintani, Molchanov, Faraut, and Strichartz among others. We summarise:

\section{Proposition 3.3.}

(1) $(p=1) V_{\lambda}^{p, q}=\{0\}$ for any $\lambda \in \mathbb{C}$.

(2) $(p \neq 1) V_{\lambda}^{p, q} \neq\{0\} \Leftrightarrow \lambda \in(p+q) / 2+2 \mathbb{Z}$ and $\lambda \neq 0$.

Furthermore, $O(p, q)$ acts irreducibly on each $V_{\lambda}^{p, q}$, when it is non-zero.

The resulting representation in Proposition 3.3 (2) will be denoted by $\pi_{\lambda}^{p, q}$. Since $V_{\lambda}^{p, q}=V_{-\lambda}^{p, q}$, we may and do assume $\operatorname{Re} \lambda \geq 0$ without loss of generality. By 
the coherent continuation of $\pi_{\lambda}^{p, q}$ for $\lambda>0$ such that $\lambda \in(p+q) / 2+2 \mathbb{Z}$, we can define irreducible unitary representations $\pi_{0}^{p, q}\left(p+q\right.$ even) and $\pi_{-1 / 2}^{p, q}(p+q$ odd $)$ of $O(p, q)$. These representations do not lie in $L^{2}\left(X_{+}^{p-1, q}\right)$ but enjoy analogous algebraic properties to $\pi_{\lambda}^{p, q}(\lambda>0)$ (see [24, §6] or [39, §5.4] for the vanishing results on cohomologies).

\section{§3.3. Quantitative branching laws}

We return to the setting of Section 2.2 The flat indefinite-Riemannian manifold $\mathbb{R}^{p, q}$ may be seen as the direct product of two flat spaces:

$$
\left(\mathbb{R}^{p}, d x_{1}^{2}+\cdots+d x_{p}^{2}\right) \times\left(\mathbb{R}^{q},-d x_{p+1}^{2}-\cdots-d x_{p+q}^{2}\right) .
$$

Likewise, the direct product of two space forms

$$
Y:=X_{+}^{p^{\prime}, q^{\prime}} \times X_{-}^{p^{\prime \prime}, q^{\prime \prime}}
$$

is locally conformal to $\mathbb{R}^{p, q}$ for any $p^{\prime}, q^{\prime}, p^{\prime \prime}, q^{\prime \prime}$ such that

$$
p^{\prime}+p^{\prime \prime}=p, \quad q^{\prime}+q^{\prime \prime}=q .
$$

This local conformal map is given as follows: For $u=\left(\left(\xi_{0}, \xi^{\prime}, \eta^{\prime}\right),\left(\xi^{\prime \prime}, \eta^{\prime \prime}, \eta_{0}\right)\right) \in$ $\mathbb{R}^{1+p^{\prime}+q^{\prime}} \oplus \mathbb{R}^{p^{\prime \prime}+q^{\prime \prime}+1}$, we set

$$
\Phi(u):=\frac{2}{\xi_{0}+\eta_{0}}\left(\xi^{\prime}, \eta^{\prime}, \xi^{\prime \prime}, \eta^{\prime \prime}\right) .
$$

Then the restriction of $\Phi$ to $Y$ is conformal (see [38, Lemma 3.3], for example). More precisely, the map

$$
\Phi: X_{+}^{p^{\prime}, q^{\prime}} \times X_{-}^{p^{\prime \prime}, q^{\prime \prime}} \rightarrow \mathbb{R}^{p, q}
$$

is well-defined and conformal in the open dense set $Y^{\prime} \subset Y$, defined by $\xi_{0}+\eta_{0} \neq 0$. Correspondingly, if we set

$$
\left(\widetilde{\Phi}^{*} f\right)(u):=\left(\frac{2}{\xi_{0}+\eta_{0}}\right)^{(p+q-2) / 2} f(\Phi(u))
$$

then $\widetilde{\Phi}^{*} f$ solves $\widetilde{\Delta}_{Y} \widetilde{\Phi}^{*} f=0$ on $Y^{\prime}$ if $\square_{p, q} f=0$ (see [38, Proposition 2.6]). Here, $\widetilde{\Delta}_{Y}$ is the Yamabe operator on $Y$, which amounts to

$$
\begin{aligned}
\widetilde{\Delta}_{Y} & =\widetilde{\Delta}_{X_{+}^{p^{\prime}, q^{\prime}}}-\widetilde{\Delta}_{X_{-}^{p^{\prime \prime}, q^{\prime \prime}}} \\
& =\Delta_{X_{+}^{p^{\prime}, q^{\prime}}}-\Delta_{X_{-}^{p^{\prime \prime}, q^{\prime \prime}}}-\frac{1}{4}\left(p^{\prime}+q^{\prime}-p^{\prime \prime}-q^{\prime \prime}\right)(p+q-2) .
\end{aligned}
$$

Hence, we can realize the minimal representation $\varpi$ of $O(p+1, q+1)$ on the solution space $\widetilde{\Delta}_{Y} F=0$ as well through $\widetilde{\Phi}^{*}$. 
We note that the map $(3.4)$ is two-to-one at generic points. In order to give a global action of the group $O(p+1, q+1)$ on the solution space to $\widetilde{\Delta}_{Y} F=0$, we need to define $F=\widetilde{\Phi}^{*} f$ by 3.5 for $\xi_{0}+\eta_{0}>0$, and by the parity condition for $\xi_{0}+\eta_{0}<0$ so that $F(-u)=(-1)^{(p-q) / 2} F(u)$ (see [40, (4.4.2a)]).

As the isometry group of $Y=X_{+}^{p^{\prime}, q^{\prime}} \times X_{-}^{p^{\prime \prime}}, q^{\prime \prime}$ is the reductive group $O\left(p^{\prime}+1, q^{\prime}\right)$ $\times O\left(p^{\prime \prime}, q^{\prime \prime}+1\right)$, it is natural to consider the branching law of the minimal representation $\varpi$ with respect to the symmetric pair

$$
O(p+1, q+1) \downarrow O\left(p^{\prime}+1, q^{\prime}\right) \times O\left(p^{\prime \prime}, q^{\prime \prime}+1\right)
$$

by using the geometric model $Y$.

In this setting, the criterion (3.1) of Theorem 3.2 holds if and only if $p^{\prime \prime}=0$ or $q^{\prime}=0$ (see [39, Theorem 4.2]). Thus, it follows from Theorem 3.2 that $\varpi$ decomposes discretely. For the description of the irreducible decomposition, we define the space of spherical harmonics of degree $l$ by

$$
\begin{aligned}
\mathcal{H}^{l}\left(\mathbb{R}^{m}\right): & =\left\{\varphi \in C^{\infty}\left(S^{m-1}\right): \Delta_{S^{m-1}} \varphi=-l(l+m-2) \varphi\right\} \\
& =\left\{\varphi \in C^{\infty}\left(S^{m-1}\right): \widetilde{\Delta}_{S^{m-1}} \varphi=\left(\frac{1}{4}-\left(l+\frac{m-2}{2}\right)^{2}\right) \varphi\right\} .
\end{aligned}
$$

The orthogonal group $O(m)$ acts irreducibly on $\mathcal{H}^{l}\left(\mathbb{R}^{m}\right)$ for any $l \in \mathbb{N}$.

Here is the branching law together with quantitative information on the invariant inner product:

Theorem 3.4 (see [39, Theorem B]). Suppose $p+q(>2)$ is even, $q=q^{\prime}+q^{\prime \prime}$, and $p, q>0$. Then the twisted pull-back $\widetilde{\Phi}^{*}$ of the conformal map $\Phi: Y \rightarrow \mathbb{R}^{p, q}$ induces the following quantitative branching law:

(1) (branching law; $\left.O(p+1, q+1) \downarrow O\left(p+1, q^{\prime}\right) \times O\left(q^{\prime \prime}+1\right)\right)$

$$
\left.\varpi^{p+1, q+1}\right|_{O\left(p+1, q^{\prime}\right) \times O\left(q^{\prime \prime}+1\right)} \simeq \sum_{l=0}^{\infty} \pi_{l+q^{\prime \prime} / 2-1 / 2}^{p+1, q^{\prime}} \otimes \mathcal{H}^{l}\left(\mathbb{R}^{q^{\prime \prime}+1}\right) .
$$

Here, the right-hand side of (3.7) is a multiplicity-free Hilbert direct sum of irreducible representations of $O\left(p+1, q^{\prime}\right) \times O\left(q^{\prime \prime}\right)$.

(2) (Parseval-type theorem) For $f \in \mathcal{S}_{0} l_{0}\left(\square_{p, q}\right)$, we expand $\widetilde{\Phi}^{*} f$ into the series $\sum_{l} F_{l}$ according to the discrete decomposition (3.7). Then

$$
\|f\|_{\mathbb{R}^{p, q}}^{2}=\sum_{l=0}^{\infty}\left(l+\frac{q^{\prime \prime}}{2}-\frac{1}{2}\right)\left\|F_{l}\right\|_{L^{2}(Y)}^{2} .
$$

Here \|\|$_{\mathbb{R}^{p, q}}$ is the norm defined in Theorem 2.6. 
In view of 3.6 , the self-adjoint operator $1 / 4-\widetilde{\Delta}_{S^{q^{\prime \prime}}}$ is non-negative, and therefore we can define a pseudo-differential operator $\left(1 / 4-\widetilde{\Delta}_{S^{q^{\prime \prime}}}\right)^{1 / 4}$ on $Y=$ $X_{+}^{p, q^{\prime}} \times S^{q^{\prime \prime}}$ as well as on $S^{q^{\prime \prime}}$.

Hence, we get another expression of the invariant inner product of the minimal representation in the geometric model $Y$ by means of a pseudo-differential operator:

Corollary 3.5. Suppose $p+q(>2)$ is even, $q=q^{\prime}+q^{\prime \prime}$, and $p, q^{\prime \prime}>0$. Let $\left(1 / 4-\widetilde{\Delta}_{S^{q^{\prime \prime}}}\right)^{1 / 4}$ be a pseudo-differential operator on $Y=X_{+}^{p, q^{\prime}} \times S^{q^{\prime \prime}}$. Set $F=\widetilde{\Phi}^{*} f$ for $f \in \overline{\mathcal{S o l}_{0}\left(\square_{p, q}\right)}$. Then

$$
\|f\|_{\mathbb{R}^{p, q}}^{2}=\left\|\left(1 / 4-\widetilde{\Delta}_{S^{q^{\prime \prime}}}\right)^{1 / 4} F\right\|_{L^{2}(Y)}^{2} .
$$

\section{Remark 3.6.}

(1) For $q^{\prime \prime}=0$ or 1 , we have $l+q^{\prime \prime} / 2-1 / 2 \leq 0$ if $l=0$. In this case $V_{l+q^{\prime \prime} / 2-1 / 2}^{p+1, q^{\prime}}=$ $\{0\}$. Nevertheless, we can justify the summand in (3.8) by using the argument of analytic continuation.

(2) In the case $p^{\prime \prime}=q^{\prime}=0$, the branching law 3.7 is nothing but the $K$-type formula for the minimal representation $\varpi$, and $(3.8)$ was proved earlier by Kostant [42] for $p=q=3$, and by Binegar and Zierau [5] for general $p, q$ such that $p+q$ is even and greater than 2 .

(3) In the case $q^{\prime \prime}=0$, we have $Y \simeq X_{+}^{p, q} \times S^{0}$, so $Y$ consists of two copies of $X_{+}^{p, q}$. Then Theorem 3.4 asserts that the minimal representation splits into two components, namely,

$$
\left.\varpi^{p+1, q+1}\right|_{O(p+1, q)} \simeq \pi_{-1 / 2}^{p+1, q} \oplus \pi_{1 / 2}^{p+1, q}
$$

because $\mathcal{H}^{l}\left(\mathbb{R}^{1}\right)=0$ for $l \geq 2$.

(4) In the case $p^{\prime \prime}=0$ and $p^{\prime}=q^{\prime}=1$, we are dealing with the branching law for the pair

$$
O(2, q+1) \downarrow O(2,1) \times O(q) .
$$

The branching law 3.7 in this special case yields a setting of the deformation of the Fourier transform (see Section 4).

\section{§4. Deformation of Fourier transforms}

Minimal representations give us also a hint to define a generalization of the Fourier transform. In this section, we introduce a holomorphic semigroup $\mathcal{I}_{k, a}(z)$ consisting of Hilbert-Schmidt operators with three parameters: 
- $a$ : interpolating minimal representations of simple groups of type C and D,

- $k$ : Dunkl deformation parameter (multiplicities on the root system),

- $z$ : complex number,

such that the operator-valued boundary value

$$
\lim _{\operatorname{Re} z \downarrow 0} \mathcal{I}_{k, a}(z)
$$

of Hilbert-Schmidt operators yields a one-parameter group of unitary operators. The underlying idea may be seen as a descendant of Sato's hyperfunction theory [47] and also that of the Gelfand-Gindikin program [11, 44, 48, for unitary representations of real reductive groups. We shall see in Diagram 4.1 that the Euclidean Fourier transform, the Hankel-type transform, the Dunkl transform, etc. arise naturally as special values of $\mathcal{I}_{k, a}(\pi i / 2)=\lim _{\varepsilon \downarrow 0} \mathcal{I}_{k, a}(\pi i / 2+\varepsilon)$.

\section{§4.1. $L^{2}$-model of minimal representations}

We return to the setting of Section 2.2. If a tempered distribution $f \in \mathcal{S}^{\prime}\left(\mathbb{R}^{p+q}\right)$ satisfies the differential equation $\square_{p, q} f=0$, then it is easy to see that its Fourier transform $\mathcal{F} f$ is supported on the characteristic variety

$$
\Xi:=\left\{\xi \in \mathbb{R}^{p+q}: \xi_{1}^{2}+\cdots+\xi_{p}^{2}-\xi_{p+1}^{2}-\cdots-\xi_{p+q}^{2}=0\right\} .
$$

What is more, the following theorem holds (see [39, Theorem 6.2]):

Theorem 4.1. For $p+q>2$ even and $p, q>0$, the Euclidean Fourier transform $\mathcal{F} \equiv \mathcal{F}_{\mathbb{R}^{p+q}}$ induces the bijection

$$
\mathcal{F}: \overline{\mathcal{S o l}_{0}\left(\square_{p, q}\right)} \stackrel{\sim}{\rightarrow} L^{2}(\Xi) .
$$

It is an isometry up to scalar multiplication by $2^{(p+q+2) / 2} \pi^{(p+q+1) / 2}$.

Here, $\overline{\mathcal{S}_{0}\left(\square_{p, q}\right)}$ is the Hilbert space with respect to the conservative quantity $\left(\right.$, ) defined in Theorem 2.6 and $L^{2}(\Xi)$ denotes the Hilbert space consisting of the square integrable functions with respect to the canonical measure on $\Xi$. The non-trivial part of Theorem 4.1 is to show that Image $\mathcal{F} \cap L^{2}(\Xi) \neq\{0\}$. See 39 , Theorem 6.2] for the proof.

It follows from Theorem 4.1 that we can realize the minimal representation of the indefinite orthogonal group $O(p+1, q+1)$ on the Hilbert space $L^{2}(\Xi)$ $\left(L^{2}\right.$-model) from the one on $\overline{\mathcal{S} o l_{0}\left(\square_{p, q}\right)}$ (conformal model).

At this moment, we remark a distinguishing feature of minimal representations (see Appendix in Section 5). Unlike well-understood families of irreducible unitary representations of real reductive groups such as unitary principal series 
representations or discrete series representations, minimal representations are so 'small' that there is no geometric model for which both group actions and the Hilbert structure are given in a simple manner (cf. [6, 50]). We write down the advantages of the aforementioned two models:

\begin{tabular}{ccc}
\hline & Group action & Hilbert structure \\
\hline Conformal model $\overline{\mathcal{S o l}\left(\square_{p, q}\right)}$ & simple & (1) \\
$L^{2}$-model $L^{2}(\Xi)$ & (2) & simple \\
\hline
\end{tabular}

Finding the missing parts (1) and (2) is interesting, particularly because of interactions with other branches of mathematics. Representation-theoretic considerations play a guiding role in formalizing problems there. In fact, we have seen in Theorem 2.6 that (1) brought us to the construction of conservative quantities for ultra-hyperbolic equations, whereas (2) leads us to the notion of a Fourier transform on the isotropic cone $\Xi$ 3, 35, 36], as discussed below.

From now on, we consider the missing part (2). In order to find a global formula for group actions on the $L^{2}$-model, let us clarify what is trivial and what will be the crucial operator. We observe that there is a maximal parabolic subgroup $P$ of $G=O(p+1, q+1)$ that contains the conformal transformation group

$$
\operatorname{Conf}\left(\mathbb{R}^{p, q}\right) \simeq\left(\mathbb{R}_{>0} \times O(p, q)\right) \ltimes \mathbb{R}^{p+q}
$$

as a subgroup of index two. Then we have the Bruhat decomposition

$$
G=P \amalg P w P, \quad \text { where } \quad w=\left(\begin{array}{cc}
I_{p+1} & 0 \\
0 & -I_{q+1}
\end{array}\right) .
$$

In fact, the Euclidean Fourier transform $\mathcal{F}_{\mathbb{R}^{N}}$ appears as the unitary inversion operator of the Segal-Shale-Weil representation of the metaplectic group $M p(N, \mathbb{R})$, which is also a minimal representation. See [37, Chapter 1] for the comparison of $\mathcal{F}_{\Xi}$ and $\mathcal{F}_{\mathbb{R}^{N}}$ from this point of view.

In the $L^{2}$-model of the minimal representation $\pi$ of $G$ on $L^{2}(\Xi)$, the $P$-action is simple: it is given just by translations and multiplications [40. Hence, it is enough to find the single unitary operator (unitary inversion operator) $\pi(w)$ in order to fill the missing part (2. We set

$$
\mathcal{F}_{\Xi}:=c \pi(w),
$$

where $c$ is the phase factor. Algebraically, $\mathcal{F}_{\Xi}$ intertwines multiplication by coordinate functions $\xi_{j}(1 \leq j \leq p+q)$ with the Bargmann-Todorov operators $R_{j}$ $(1 \leq j \leq p+q)$ which are mutually commuting differential operators of second order on $\Xi$ (see [2], 36, Chapter 1]). 
This algebraic feature is similar to the classical fact that the Euclidean Fourier transform $\mathcal{F}_{\mathbb{R}^{N}}$ intertwines the multiplication operators $\xi_{j}$ and the differential operators $\sqrt{-1} \partial_{j}(1 \leq j \leq N)$.

The goal of this section is to explain these operators $\mathcal{F}_{\Xi}$ and $\mathcal{F}_{\mathbb{R}^{N}}$ from a broader point of view, by constructing a continuous family of operators that includes $\mathcal{F}_{\Xi}$ and $\mathcal{F}_{\mathbb{R}^{N}}$.

For this, we limit ourselves to the case $p=1$. Then the light cone $\Xi$ (see (4.1p) splits into the forward light cone $\Xi_{+}$and the backward light cone $\Xi_{-}$according as $x_{1}>0$ and $x_{1}<0$. The unitary inversion operator $\mathcal{F}_{\Xi}$ preserves the direct sum

$$
L^{2}(\Xi)=L^{2}\left(\Xi_{+}\right) \oplus L^{2}\left(\Xi_{-}\right) .
$$

For later purpose, we set $q=N$. Then the projection to the second factor, $\mathbb{R}^{1} \oplus \mathbb{R}^{N} \rightarrow \mathbb{R}^{N}$, induces the following isomorphism between Hilbert spaces:

$$
L^{2}\left(\Xi_{+}\right) \simeq L^{2}\left(\mathbb{R}^{N},\|x\|^{-1} d x\right) .
$$

Via 4.4, the unitary inversion operator $\mathcal{F}_{\Xi}$ on $L^{2}\left(\Xi_{+}\right)$may be seen as a unitary operator on $L^{2}\left(\mathbb{R}^{N},\|x\|^{-1} d x\right)$. The explicit formula for $\mathcal{F}_{\Xi}$ in the coordinates of $\mathbb{R}^{N}$ was given in [35. In this framework, we can construct a holomorphic family of bounded operators so that the unitary operator $\mathcal{F}_{\Xi}$ is obtained as the limit of holomorphic objects. Deformation in the Dunkl setting [3, 4] is also built on this formulation. We will discuss those operators in this generality in Section 4.4

An alternative approach was taken in [36] based on the Barnes-Mellin integral to find the kernel function of $\mathcal{F}_{\Xi}$ for general $p$ and $q$.

\section{§4.2. Hermite semigroup and Fourier transform}

We begin by recalling that the classical Hermite operator on $\mathbb{R}^{N}$ (e.g. 9, 18])

$$
\Delta-\|x\|^{2}=\sum_{j=1}^{N} \frac{\partial^{2}}{\partial x_{j}^{2}}-\sum_{j=1}^{N} x_{j}^{2}
$$

extends to a self-adjoint operator on $L^{2}\left(\mathbb{R}^{N}\right)$. We normalize the Euclidean Fourier transform $\mathcal{F}_{\mathbb{R}^{N}}$ on $L^{2}\left(\mathbb{R}^{N}\right)$ as

$$
\left(\mathcal{F}_{\mathbb{R}^{N}} f\right)(\xi)=\frac{1}{(2 \pi)^{N / 2}} \int_{\mathbb{R}^{N}} f(x) e^{-i\langle x, \xi\rangle} d x .
$$

Then $\mathcal{F}_{\mathbb{R}^{N}}$ is a special value of the one-parameter group of unitary operators

$$
\chi(t):=\exp \left(\frac{i t}{2}\left(\Delta-\|x\|^{2}\right)\right),
$$


namely, we have

$$
\mathcal{F}_{\mathbb{R}^{N}}=e^{\frac{1}{4} \pi i N} \exp \left(\frac{\pi i}{4}\left(\Delta-\|x\|^{2}\right)\right) .
$$

Further, the one-parameter group $\chi(t)$ of unitary operators extends to a holomorphic semigroup $I(z)$ defined by

$$
I(z)=\exp \left(\frac{z}{2}\left(\Delta-\|x\|^{2}\right)\right) \quad \text { for } \operatorname{Re} z>0 .
$$

The semigroup $I(z)$ is called the Hermite semigroup, and it is expressed as an integral transform against the Mehler kernel [9, 18], a Gaussian type kernel.

Next, we consider another differential operator on $\mathbb{R}^{N}$,

$$
\|x\| \Delta-\|x\| \text {. }
$$

It turns out that this operator has a self-adjoint extension on the Hilbert space $L^{2}\left(\mathbb{R}^{N},\|x\|^{-1} d x\right)$ (see [35, Section 1.1]). Moreover, an analogous formula to 4.6 holds: via the identification (4.4), the 'Fourier transform' $\mathcal{F}_{\Xi}$ on the forward light cone $\Xi_{+}$can be expressed as

$$
\mathcal{F}_{\Xi}=c \exp \left(\frac{\pi i}{2}(\|x\| \Delta-\|x\|)\right)
$$

where $c=e^{\frac{1}{2} \pi i(N-1)}$ is the phase factor. The expression 4.8 allows us to see $\mathcal{F}_{\Xi}$ as the limit of the following holomorphic semigroup (Laguerre semigroup):

$$
\mathcal{I}(z)=\exp (z(\|x\| \Delta-\|x\|)) \quad \text { for } \operatorname{Re} z>0,
$$

as $z \rightarrow \pi i / 2+0$. The kernel function of $\mathcal{I}(z)$ is given in terms of the Bessel function 33 .

Interpolating $\Delta-\|x\|^{2}$ and $\|x\| \Delta-\|x\|$, the infinitesimal generators of the Hermite semigroup (4.7) and the Laguerre semigroup 4.9 , we consider the differential operator

$$
\Delta_{0, a}:=\|x\|^{2-a} \Delta-\|x\|^{a} .
$$

It might not be obvious that the symmetric operator $\Delta_{0, a}$ has a self-adjoint extension on the Hilbert space $L^{2}\left(\mathbb{R}^{N},\|x\|^{a-2} d x\right)$. In fact, this is the case. The proof uses representation theory (see Proposition 4.6), and the same idea works in a more general setting of Dunkl's differential-difference operators. Thus, in Section 4.3 we shall introduce a holomorphic semigroup $\mathcal{I}_{k, a}(z)$ with infinitesimal generator $\Delta_{k, a}$ (see 4.10 below for the definition) for $\operatorname{Re} z>0$ with parameters $k$ and $a$.

In Diagram 4.1, we have summarised some of the deformation properties by indicating the limit behavior of the holomorphic semigroup $\mathcal{I}_{k, a}(z)$. The specialization $\mathcal{I}_{k, a}(\pi i / 2)$ leads us to a $(k, a)$-generalized Fourier transform $\mathcal{F}_{k, a}$ (up to 
a phase factor), which reduces to the Fourier transform $(a=2$ and $k \equiv 0)$, the Dunkl transform $\mathcal{D}_{k}(a=2$ and $k \not \equiv 0)$, and the Hankel-type transform $(a=1$ and $k \equiv 0)$.

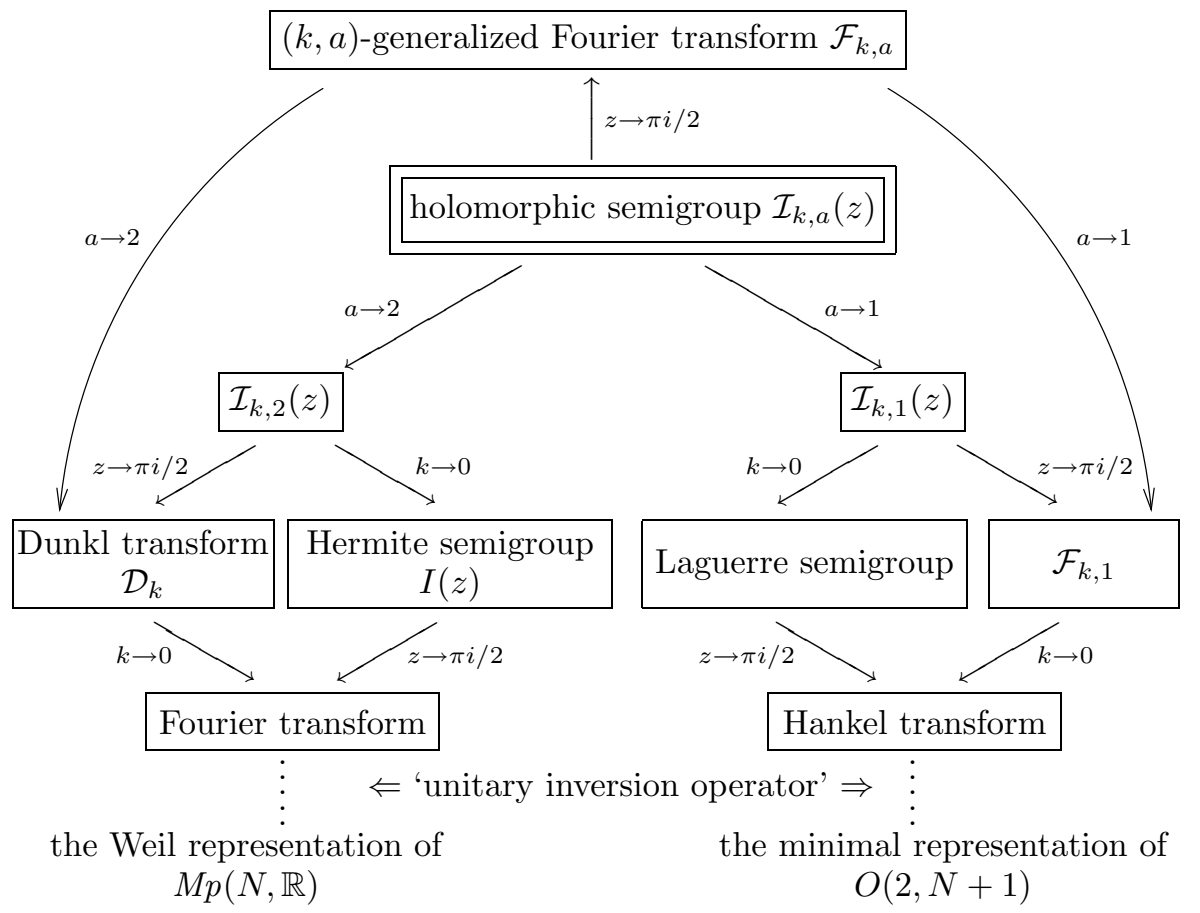

Diagram 4.1. Special values of the holomorphic semigroup $\mathcal{I}_{k, a}(z)$.

\section{§4.3. Holomorphic semigroup $\mathcal{I}_{k, a}(z)$ with two parameters $k$ and $a$}

This subsection introduces a holomorphic semigroup, denoted by $\mathcal{I}_{k, a}(z)$, whose infinitesimal generator is a self-adjoint differential-difference operator.

Let $\mathfrak{C}$ be the Coxeter group associated with a reduced root system $\mathcal{R}$ in $\mathbb{R}^{N}$. For a $\mathfrak{C}$-invariant function $k \equiv\left(k_{\alpha}\right)$ (multiplicity function) on $\mathcal{R}$, we set

$$
\langle k\rangle:=\frac{1}{2} \sum_{\alpha \in \mathcal{R}} k_{\alpha},
$$

and write $\Delta_{k}$ for the Dunkl Laplacian on $\mathbb{R}^{N}$ (see [15]). This is a differentialdifference operator, which reduces to the Euclidean Laplacian $\Delta$ when $k \equiv 0$.

We take $a>0$ to be yet another deformation parameter, and define

$$
\Delta_{k, a}:=\|x\|^{2-a} \Delta_{k}-\|x\|^{a} .
$$


We define a density on $\mathbb{R}^{N}$ by

$$
\vartheta_{k, a}(x):=\|x\|^{a-2} \prod_{\alpha \in \mathcal{R}}|\langle\alpha, x\rangle|^{k_{\alpha}}
$$

The volume of the unit ball with respect to the measure $\vartheta_{k, a}(x) d x$ is explicitly known in terms of the gamma function owing to the work by Selberg, Macdonald, Heckman, and Opdam among others but we do not go into details (see Etingof [8]).

In the case $a=2$ and $k \equiv 0, \vartheta_{0,2}(x) \equiv 1$ and $\Delta_{0,2}$ is the Hermite operator (4.5) on $\mathbb{R}^{N}$.

Here are the basic properties of our differential-difference operator $\Delta_{k, a}$ :

Theorem 4.2 (see [4, Theorem A]). Assume $a>0$ and $a+2\langle k\rangle+N-2>0$.

(1) $\Delta_{k, a}$ extends to a self-adjoint operator on the Hilbert space $L^{2}\left(\mathbb{R}^{N}, \vartheta_{k, a}(x) d x\right)$.

(2) $\Delta_{k, a}$ has no continuous spectrum.

(3) All the discrete spectrum of $\Delta_{k, a}$ is negative.

We introduce the following operators on $L^{2}\left(\mathbb{R}^{N}, \vartheta_{k, a}(x) d x\right)$ :

$$
\mathcal{I}_{k, a}(z):=\exp \left(\frac{z}{a} \Delta_{k, a}\right) \quad \text { for } \operatorname{Re} z \geq 0
$$

Correspondingly to the properties of the infinitesimal generator $\frac{1}{a} \Delta_{k, a}$ in Theorem 4.2 , we get:

Theorem 4.3 (see [4, Theorem B]). Retain the assumption of Theorem 4.2.

(1) $\left\{\mathcal{I}_{k, a}(z): \operatorname{Re} z>0\right\}$ forms a holomorphic semigroup in the complex righthalf plane $\{z \in \mathbb{C}: \operatorname{Re} z>0\}$ in the sense that $\mathcal{I}_{k, a}(z)$ is a Hilbert-Schmidt operator on $L^{2}\left(\mathbb{R}^{N}, \vartheta_{k, a}(x) d x\right)$ satisfying

$$
\mathcal{I}_{k, a}\left(z_{1}\right) \circ \mathcal{I}_{k, a}\left(z_{2}\right)=\mathcal{I}_{k, a}\left(z_{1}+z_{2}\right) \quad\left(\operatorname{Re} z_{1}, \operatorname{Re} z_{2}>0\right),
$$

and the scalar product $\left(\mathcal{I}_{k, a}(z) f, g\right)$ is a holomorphic function of $z$ for $\operatorname{Re} z>0$, for any $f, g \in L^{2}\left(\mathbb{R}^{N}, \vartheta_{k, a}(x) d x\right)$.

(2) $\mathcal{I}_{k, a}(z)$ is a one-parameter group of unitary operators on the imaginary axis $\operatorname{Re} z=0$.

We shall call $\mathcal{I}_{k, a}(z)$ the $(k, a)$-generalized Laguerre semigroup. We note that $\mathcal{I}_{0,2}(z)$ is the Hermite semigroup 4.7) (see [9, 18]), and $\mathcal{I}_{0,1}(z)$ is the Laguerre semigroup 4.9) (see 33]). 
§4.4. $(k, a)$-generalized Fourier transforms $\mathcal{F}_{k, a}$

Theorem 4.3(2) asserts that the 'boundary value' of the holomorphic semigroup $\mathcal{I}_{k, a}(z)$ produces a one-parameter family of unitary operators.

The case $z=0$ gives the identity operator, $\mathcal{I}_{k, a}(0)=$ id. A particularly interesting case is when $z=\pi i / 2$. We set

$$
c:=\exp \left(i \pi \frac{N+2\langle k\rangle+a-2}{2 a}\right) \quad \text { (phase factor) }
$$

and define the $(k, a)$-generalized Fourier transform by

$$
\mathcal{F}_{k, a}:=c \mathcal{I}_{k, a}\left(\frac{\pi i}{2}\right)=c \exp \left(\frac{\pi i}{2 a}\left(\|x\|^{2-a} \Delta_{k}-\|x\|^{a}\right)\right) .
$$

The operator $\mathcal{F}_{k, a}$ for general $a$ and $k$ has the following significant properties:

Theorem 4.4 (4, Theorem D]). Retain the assumption of Theorem 4.2 ,

(1) $\mathcal{F}_{k, a}$ is a unitary operator on $L^{2}\left(\mathbb{R}^{N}, \vartheta_{k, a}(x) d x\right)$.

(2) $\mathcal{F}_{k, a} \circ H_{k, a}=-H_{k, a} \circ \mathcal{F}_{k, a}$, where $H_{k, a}$ is the differential operator of first order defined in 4.12 below.

(3) $\mathcal{F}_{k, a} \circ\|x\|^{a}=-\|x\|^{2-a} \Delta_{k} \circ \mathcal{F}_{k, a}$ and $\mathcal{F}_{k, a} \circ\left(\|x\|^{2-a} \Delta_{k}\right)=-\|x\|^{a} \circ \mathcal{F}_{k, a}$.

(4) $\mathcal{F}_{k, a}$ is of finite order if and only if $a \in \mathbb{Q}$. Its order is $2 m$ if $a=m / n$ with $(m, n)=1$. In particular, $\mathcal{F}_{k, 1}$ is of order 2 , and $\mathcal{F}_{k, 2}$ is of order 4.

As indicated in Diagram 4.1, $\mathcal{F}_{k, a}$ reduces to the Euclidean Fourier transform $\mathcal{F}$ on $\mathbb{R}^{N}$ if $k \equiv 0$ and $a=2$; and to the Dunkl transform $\mathcal{D}_{k}$ introduced by C. Dunkl himself if $k>0$ and $a=2$. The unitary operator $\mathcal{F}_{0,1}$ arises as the unitary inversion operator $\mathcal{F}_{\Xi}$ on $L^{2}\left(\Xi_{+}\right)$of the minimal representation of the conformal group (see Section 4.1).

Our study also contributes to the theory of special functions, in particular orthogonal polynomials; indeed we derive several new identities, for example, the $(k, a)$-deformation of the classical Bochner-Hecke identity where the Gaussian function and harmonic polynomials in the classical setting $(k \equiv 0$ and $a=2)$ are replaced respectively with $\exp \left(-\frac{1}{a}\|x\|^{a}\right)$ and polynomials annihilated by the Dunkl Laplacian. The $(k, a)$-generalized Fourier transform $\mathcal{F}_{k, a}$ also satisfies a Heisenberg-type inequality. This generalizes the classical case ( $k \equiv 0$ and $a=2)$ and Rösler's Heisenberg inequality [45] ( $k>0$ and $a=2)$. We refer to [4] for full details. 


\section{§4.5. Hidden symmetries in the Hilbert space $L^{2}\left(\mathbb{R}^{N}, \vartheta_{k, a}(x) d x\right)$}

The key idea of the proofs of Theorem 4.14 .3 is to use more operators rather than the single operator $\Delta_{k, a}$, and then to appeal to the representation theory of $\mathfrak{s l}_{2}$, in particular, the theory of discretely decomposable unitary representations.

Lemma 4.5. Let $k$ be a multiplicity function on a root system, and $a \in \mathbb{C}^{\times}$. Then the differential-difference operators on $\mathbb{R}^{N} \backslash\{0\}$

$$
\begin{aligned}
E_{k, a}^{+} & :=\frac{i}{a}\|x\|^{a}, \\
E_{k, a}^{-} & :=\frac{i}{a}\|x\|^{2-a} \Delta_{k}, \\
H_{k, a} & :=\frac{2}{a} \sum_{i=1}^{N} x_{i} \partial_{i}+\frac{N+2\langle k\rangle+a-2}{a}
\end{aligned}
$$

form an $\mathfrak{s l}_{2}$-triple, namely,

$$
\left[H_{k, a}, E_{k, a}^{+}\right]=2 E_{k, a}^{+}, \quad\left[H_{k, a}, E_{k, a}^{-}\right]=-2 E_{k, a}^{-}, \quad\left[E_{k, a}^{+}, E_{k, a}^{-}\right]=H_{k, a} .
$$

Special cases of Lemma 4.5 were previously known: the case $k \equiv 0$ and $a=2$ is the classical harmonic $\mathfrak{s l}_{2}$-triple (e.g. Howe [18]), the case $k>0$ and $a=2$ is due to Heckman [15], and $k \equiv 0$ and $a=1$ to Kobayashi and Mano [33. The operator $\Delta_{k, a}($ see 4.10$)$ ) takes the form

$$
\Delta_{k, a}=a i\left(E_{k, a}^{+}-E_{k, a}^{-}\right),
$$

which may be seen as an element of $\mathfrak{s l}(2, \mathbb{C})$.

Lemma 4.5 fits well into the framework of discretely decomposable representations of reductive groups [25 27] as discussed in Section 3.1.

Theorem 4.6 (see [4, Theorem 3.31]). If $a>0$ and $a+2\langle k\rangle+N-2>0$, then the representation of $\mathfrak{s l}(2, \mathbb{R})$ lifts to a unitary representation of the simply-connected group on $L^{2}\left(\mathbb{R}^{N}, \vartheta_{k, a}(x) d x\right)$. The resulting unitary representation is discretely decomposable, and commutes with the obvious action of the Coxeter group $\mathfrak{C}$.

This unitary representation plays a central role in the proof of Theorems 4.2 4.4. An explicit formula for the irreducible decomposition of $L^{2}\left(\mathbb{R}^{N}, \vartheta_{k, a}(x)\right)$ is found in [4, Theorem 3.28]. In the special cases $k \equiv 0$ and $a=1$ or 2 , this formula may be regarded as the branching law of the minimal representations of $O(2, N+1) \sim$ or $M p(N, \mathbb{R})$, respectively (see Diagram 4.2 below). Correspondingly, all the spectrum of $\Delta_{k, a}$ is also obtained explicitly.

In the case $a=2$, the $\mathfrak{s l}_{2}$-action also induces a representation of $S L(2, \mathbb{C})$ on the algebra generated by Dunkl's operators, multiplication operators, and the 
Coxeter group. The restriction of this action to $S L(2, \mathbb{Z})$ coincides with a special case of the $S L(2, \mathbb{Z})$-action discovered by Cherednik [7] on the (degenerate) rational DAHA (double affine Hecke algebra).

Theorem 4.6 asserts that the Hilbert space $L^{2}\left(\mathbb{R}^{N}, \vartheta_{k, a}(x) d x\right)$ has a symmetry of the direct product group $\mathfrak{C} \times \widehat{S L(2, \mathbb{R})}$ for all $k$ and $a$. This symmetry becomes larger for special values of $k$ and $a$ :

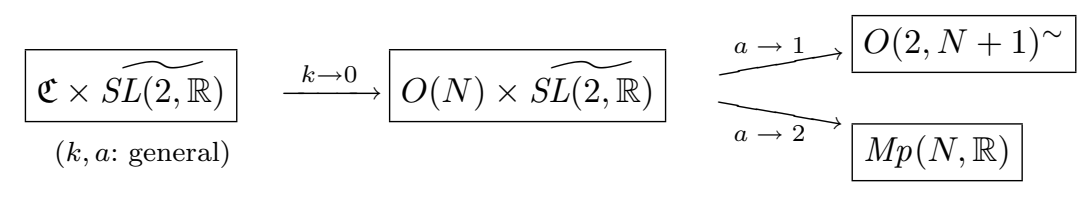

Diagram 4.2. Hidden symmetries in $L^{2}\left(\mathbb{R}^{N}, \vartheta_{k, a}(x) d x\right)$.

For $a=2$, this symmetry is given by the Segal-Shale-Weil representation of the metaplectic group $M p(N, \mathbb{R})$. For $a=1$, it is given by the irreducible unitary representation of the double covering $O(2, N+1)^{\sim}$ of the conformal group on $L^{2}\left(\mathbb{R}^{N},\|x\|^{-1} d x\right)$. Here, as we saw in Theorem 4.1 , we do not need to take a double cover when $N$ is odd. Both of them are minimal representations and, in particular, they attain the minimum of their Gelfand-Kirillov dimensions among the unitary dual. In this sense, our continuous parameter $a>0$ interpolates the $L^{2}$-models of two minimal representations of different reductive groups by keeping smaller symmetries $O(N) \times \widehat{S L(2, \mathbb{R})}$. In view of Lemma 4.5 , the $(k, a)$-generalized Fourier transform $\mathcal{F}_{k, a}(k \equiv 0, a=1,2)$ arises as the unitary operator (up to a phase factor) corresponding to

$$
\exp \left(\frac{\pi}{2}\left(\begin{array}{cc}
0 & 1 \\
-1 & 0
\end{array}\right)\right) \in \widetilde{S L(2, \mathbb{R})}
$$

\section{$\S 5$. Appendix: representation-theoretic properties of $\varpi$}

For the reader's convenience, we list some representation-theoretic properties of the irreducible unitary representation $\varpi$ of the indefinite orthogonal group $G=$ $O(p+1, q+1)$, whose geometric analysis is the leading theme of this article.

In what follows, we assume

$p, q \geq 1$ and $p+q$ is an even integer greater than two.

We write $K \simeq O(p+1) \times O(q+1)$ for a maximal compact group of $G, \mathfrak{g} \simeq$ $\mathfrak{o}(p+1, q+1)$ for the Lie algebra of $G, \mathfrak{g}_{\mathbb{C}} \simeq \mathfrak{o}(p+q+2, \mathbb{C})$ for its complexification, and $\mathfrak{g}_{\mathbb{C}}=\mathfrak{k}_{\mathbb{C}}+\mathfrak{p}_{\mathbb{C}}$ for the complexified Cartan decomposition. 
Here are some properties of $\varpi$ from representation-theoretic viewpoints.

(1) $\varpi$ is an irreducible unitary representation of $G$.

(2) (Minimal representation) The representation $\varpi$ is a minimal representation in the sense that the annihilator of the underlying $\left(\mathfrak{g}_{\mathbb{C}}, K\right)$-module $\varpi_{K}$ in the universal enveloping algebra $U\left(\mathfrak{g}_{\mathbb{C}}\right)$ is the Joseph ideal if $p+q \geq 6([5,42])$. See [10] for algebraic aspects of minimal representations of reductive groups and the definition of the Joseph ideal.

(3) (Restriction to the identity component) The group $G$ has four connected components, and we write $G_{0}=S O_{0}(p+1, q+1)$ for the identity component; $\varpi$ stays irreducible when restricted to $G_{0}$ if and only if $p, q>1$.

(4) (Highest weight module case) If $p=1$ or $q=1$, then the restriction $\left.\varpi\right|_{G_{0}}$ is a direct sum of two irreducible representations; one is a highest weight representation $\varpi_{+}$and the other is a lowest weight representation $\varpi_{-}$. As we have seen in $(4.3)$, this decomposition $\left.\varpi\right|_{G_{0}}=\varpi_{+} \oplus \varpi_{-}$corresponds to the direct sum

$$
L^{2}(\Xi)=L^{2}\left(\Xi_{+}\right) \oplus L^{2}\left(\Xi_{-}\right)
$$

in the $L^{2}$-model. Both $\varpi_{+}$and $\varpi_{-}$are minimal representations of the connected group $G_{0}$.

To fix notation, we suppose $p=1$. Then $G$ is the conformal group $O(2, q+1)$ of the Minkowski space $\mathbb{R}^{1, q}$, the Euclidean space $\mathbb{R}^{1+q}$ equipped with the flat Lorentz metric of signature $(1, q)$. In this case our representation $\varpi$ has a long history of study, also in physics (see e.g. Todorov [49]). The minimal representation $\varpi_{+}$may be interpreted as the symmetry of the solution space to the mass-zero spin-zero wave equation. The representation $\varpi_{+}$also arises on the Hilbert space of bound states of the hydrogen atom.

(5) (Spherical case) The underlying $\left(\mathfrak{g}_{\mathbb{C}}, K\right)$-module $\varpi_{K}$ has the following $K$-type formula:

$$
\varpi_{K} \simeq \bigoplus_{\substack{a, b \in \mathbb{N}, a+p / 2=b+q / 2}} \mathcal{H}^{a}\left(\mathbb{R}^{p+1}\right) \otimes \mathcal{H}^{b}\left(\mathbb{R}^{q+1}\right)
$$

In particular, the representation $\varpi$ is spherical (i.e. contains a non-zero $K$-fixed vector) if and only if $p=q$.

(6) (Infinitesimal character) Let $\mathfrak{Z}\left(\mathfrak{g}_{\mathbb{C}}\right)$ be the center of $U\left(\mathfrak{g}_{\mathbb{C}}\right)$. Then the infinitesimal character of $\varpi_{K}$ is given by

$$
\left(1, \frac{p+q}{2}-1, \frac{p+q}{2}-2, \ldots, 1,0\right) .
$$


Here, we normalize the Harish-Chandra isomorphism for the simple Lie algebra $\mathfrak{g}_{\mathbb{C}}$ of type $D_{n}(n=(p+q) / 2+1)$,

$$
\operatorname{Hom}_{\mathbb{C} \text {-algebra }}\left(\mathfrak{Z}\left(\mathfrak{g}_{\mathbb{C}}\right), \mathbb{C}\right) \simeq \mathbb{C}^{n} / W\left(D_{n}\right)
$$

that the infinitesimal character of the trivial one-dimensional representation is

$$
\left(\frac{p+q}{2}, \frac{p+q}{2}-1, \frac{p+q}{2}-2, \ldots, 1,0\right) .
$$

(7) (Theta correspondence) The representation $\varpi$ is obtained also as the theta correspondence of the trivial one-dimensional representation of $S L(2, \mathbb{R})$ with respect to the reductive dual pair

$$
O(p+1, q+1) \cdot S L(2, \mathbb{R}) \subset S p(p+q+2, \mathbb{R}) .
$$

See 52 .

(8) (Gelfand-Kirillov dimension) The Gelfand-Kirillov dimension of the representation $\varpi$, denoted by $\operatorname{DIM}(\varpi)$, attains its minimum among all unitary representations of $G$, that is,

$$
\operatorname{DIM}(\varpi)=p+q-1 .
$$

The associated variety of the underlying $\left(\mathfrak{g}_{\mathbb{C}}, K\right)$-module $\varpi_{K}$ is given by

$$
\operatorname{AV}(\varpi)=\mathcal{O}_{\min }^{\mathbb{C}} \cap \mathfrak{p}_{\mathbb{C}}
$$

(see [39, Lemma 4.4]). Here, $\mathcal{O}_{\text {min }}^{\mathbb{C}}$ is the minimal nilpotent coadjoint orbit in $\mathfrak{g}_{\mathbb{C}}^{*}$ identified with the Lie algebra $\mathfrak{g}_{\mathbb{C}}$.

\section{Acknowledgements}

This research was partially supported by Grant-in-Aid for Scientific Research (B) (18340037), Japan Society for the Promotion of Science.

\section{References}

[1] M. F. Atiyah, The Harish-Chandra character, in Representation theory of Lie groups (Oxford, 1977), London Math. Soc. Lecture Note Ser. 34, Cambridge Univ. Press, 1979, 176-181. Zbl 0426.22014

[2] V. Bargmann and I. T. Todorov, Spaces of analytic functions on a complex cone as carriers for the symmetric tensor representations of $S O(n)$, J. Math. Phys. 18 (1977), 1141-1148. Zbl 0364.46016 MR 0486330

[3] S. Ben Saïd, T. Kobayashi, and B. Ørsted, Generalized Fourier transforms $\mathcal{F}_{k, a}$ C. R. Math. Acad. Sci. Paris 347 (2009), 1119-1124. Zbl 1176.43003 MR 2566988

[4] _ Laguerre semigroup and Dunkl operators, arXiv:0907.3749, 74 pp. 
[5] B. Binegar and R. Zierau, Unitarization of a singular representation of $S O(p, q)$, Comm. Math. Phys. 138 (1991), 245-258. Zbl 0748.22009 MR 1108044

[6] R. Brylinski and B. Kostant, Differential operators on conical Lagrangian manifolds, in Lie theory and geometry, Progr. Math. 123, Birkhäuser, Boston, 1994, 65-96. Zbl 0878.58033 MR 1327531

[7] I. Cherednik, Macdonald's evaluation conjectures and difference Fourier transform, Invent. Math. 122 (1995), 119-145. Zbl 0854.22021 MR 1354956

[8] P. Etingof, A uniform proof of the Macdonald-Mehta-Opdam identity for finite Coxeter groups, Math. Res. Lett. 17 (2010), 275-282. MR 2644375

[9] G. B. Folland, Harmonic analysis in phase space, Ann. of Math. Stud. 122, Princeton Univ. Press, Princeton, NJ, 1989. Zbl 0682.43001 MR 0983366

[10] W. T. Gan and G. Savin, On minimal representations definitions and properties, Represent. Theory 9 (2005), 46-93. Zbl 1092.22012 MR 2123125

[11] I. M. Gelfand and S. G. Gindikin, Complex manifolds whose spanning trees are real semisimple Lie groups, and analytic discrete series representations, Funktsional. Anal. i Prilozhen. 11 (1977), no. 4, 19-27, 96 (in Russian). Zbl 0449.22018 Zbl 0444.22006 MR 0492076

[12] B. H. Gross and N. R. Wallach, A distinguished family of unitary representations for the exceptional groups of real rank $=4$, in Lie theory and geometry, Progr. Math. 123, Birkhäuser Boston, Boston, MA, 1994, 289-304. Zbl 0839.22006 MR 1327538

[13] S. Hansen, J. Hilgert, and S. Keliny, Asymptotic $K$-support and restrictions of representations Represent. Theory 13 (2009), 460-469. Zbl 1202.22016 MR 2550473

[14] Harish-Chandra, Representations of semisimple Lie groups, I, III, Trans. Amer. Math. Soc. 75 (1953), 185-243; 76 (1954), 234-253; IV, Amer. J. Math. 77 (1955), 743777. Zbl 0051.34002(I) Zbl 0055.34002(III) Zbl 0066.35603(IV) MR 0056610(I) MR 0062747(III) MR 0072427(IV)

[15] G. J. Heckman, A remark on the Dunkl differential-difference operators, in Harmonic analysis on reductive groups, W. Barker and P. Sally (eds.), Progr. Math. 101, Birkhäuser, 1991, 181-191. Zbl 0749.33005 MR 1168482

[16] J. Hilgert, T. Kobayashi, G. Mano, and J. Möllers, Special functions associated to a certain fourth order differential equation, Ramanujan J., to appear; arXiv:0907.2608, 31 pp.

[17] _ Orthogonal polynomials associated to a certain fourth order differential equation, Ramanujan J., to appear; arXiv:0907.2612.

[18] R. Howe, The oscillator semigroup, in The mathematical heritage of Hermann Weyl (Durham, NC, 1987), R. O. Wells, Jr. (ed.), Proc. Sympos. Pure Math. 48, Amer. Math. Soc., 1988, 61-132. Zbl 0687.47034 MR 0974332

[19] R. Howe and E.-C. Tan, Homemogeneous functions on light cones: the infinitesimal structure of some degenerate principal series representations, Bull. Amer. Math. Soc. 28 (1993), 1-74. Zbl 0794.22012 MR 1172839

[20] M. Kashiwara, T. Kawai, and T. Kimura, Foundations of algebraic analysis, Princeton Math. Ser. 37, Princeton Univ. Press, 1986. Zbl 0605.35001 MR 0855641

[21] M. Kashiwara and M. Vergne, K-types and singular spectrum, in Noncommutative harmonic analysis (Marseille-Luminy, 1978), Lecture Notes in Math. 728, Springer, 1979, 177-200. Zbl 0411.22015 MR 0548330

[22] D. Kazhdan, The minimal representation of $D_{4}$, in Operator algebras, unitary representations, enveloping algebras, and invariant theory (Paris, 1989), Progr. Math. 92, Birkhäuser, Boston, 1990, 125-158. Zbl 0781.22013 MR 1103589

[23] D. Kazhdan and G. Savin, The smallest representation of simply laced groups, in Festschrift in honor of I. I. Piatetski-Shapiro on the occasion of his sixtieth birthday, Part I (Ramat Aviv, 1989), Israel Math. Conf. Proc. 2, Weizmann, Jerusalem, 1990, 209-223. Zbl 0737.22008 MR 1159103 
[24] T. Kobayashi, The restriction of $A_{\mathfrak{q}}(\lambda)$ to reductive subgroups, Proc. Japan Acad. 69 (1993), 262-267. Zbl 0826.22014 MR 1249224

[25] Discrete decomposability of the restriction of $A_{\mathfrak{q}}(\lambda)$ with respect to reductive subgroups and its application, Invent. Math. 117 (1994), 181-205. Zbl 0826.22015 MR 1273263

[26] Discrete decomposability of the restriction of $A_{\mathfrak{q}}(\lambda)$, II. Micro-local analysis and asymptotic $K$-support, Ann. of Math. 147 (1998), 709-729. Zbl 0910.22016 MR 1637667

[27] Discrete decomposability of the restriction of $A_{\mathfrak{q}}(\lambda)$, III. Restriction of Harish-Chandra modules and associated varieties, Invent. Math. 131 (1998), 229-256. Zbl 0907.22016 MR 1608642

[28] _ Discretely decomposable restrictions of unitary representations of reductive Lie groups - examples and conjectures, in Analysis on homogeneous spaces and representation theory of Lie groups (Okayama-Kyoto, 1997), Adv. Stud. Pure Math. 26, Math. Soc. Japan, 2000, 99-127. Zbl 0959.22009 MR 1770719

[29] Branching problems of unitary representations in Proc. ICM 2002 (Beijing), Vol. 2, 2002, 615-627. Zbl 1008.43009 MR 1957069

[30] Conformal geometry and global solutions to the Yamabe equations on classical pseudo-Riemannian manifolds, in Proc. 22nd Winter School "Geometry and Physics" (Srní, 2002), Rend. Circ. Mat. Palermo (2) Suppl. 71 ( 2003), 15-40. Zbl 1074.53031 MR 1982432

[31] Theory of discretely decomposable restrictions of unitary representations of semisimple Lie groups and some applications, Sugaku Expositions 18 (2005), 1-37. Zbl pre05509226 MR 2132098

[32] Restrictions of unitary representations of real reductive groups in Lie theory, Progr. Math. 229, Birkhäuser, 2005, 139-207. |Zbl 1072.22008||MR 2126642

[33] T. Kobayashi and G. Mano, Integral formulas for the minimal representation of $O(p, 2)$, Acta Appl. Math. 86 (2005), 103-113. Zbl 1100.22007|| MR 2134314

[34] _ Integral formula of the unitary inversion operator for the minimal representation of $O(p, q)$ Proc. Japan Acad. Ser. A Math. Sci. 83 (2007), 27-31. Zbl pre05195226 MR 2317306

[35] T. Kobayashi and G. Mano, The inversion formula and holomorphic extension of the minimal representation of the conformal group, in Harmonic analysis, group representations, automorphic forms and invariant theory: in honor of Roger E. Howe (J.-S. Li et al., eds.), Singapore Univ. Press and World Sci., 2007, 159-223.

[36] T. Kobayashi and B. Ørsted, The Schrödinger model for the minimal representation of the indefinite orthogonal group $O(p, q)$, Mem. Amer. Math. Soc. 213 (2011), no. 1000.

[37] , Conformal geometry and branching laws for unitary representations attached to minimal nilpotent orbits, C. R. Acad. Sci. Paris 326 (1998), 925-930. Zbl 0910.22010 MR 1649917

[38] _ Analysis on the minimal representation of $O(p, q)$. I. Realization via conformal geometry, Adv. Math. 180 (2003), 486-512. Zbl 1046.22004 MR 2020550

[39] Analysis on the minimal representation of $O(p, q)$. II. Branching laws Adv. Math. 180 (2003), 513-550. Zbl 1049.22006 $\mid$ MR 2020551

[40] Analysis on the minimal representation of $O(p, q)$. III. Ultrahyperbolic equations \begin{tabular}{|l|l|l|l|}
\hline on $\mathbb{R}^{p-1, q-1}$ & Adv. Math. 180 (2003), 551-595. & Zbl 1039.22005 & MR 2020552 \\
\hline
\end{tabular}

[41] T. Kobayashi, B. Ørsted and M. Pevzner, Geometric analysis on small unitary representations of $G L(N, \mathbb{R})$, J. Funct. Anal. 260 (2011), 1682-1720. Zbl pre05868407 MR 2754889

[42] B. Kostant, The vanishing of scalar curvature and the minimal representation of $S O(4,4)$, in Operator algebras, unitary representations, enveloping algebras, and invariant theory (Paris, 1989), A. Connes et al. (eds.), Progr. Math. 92, Birkhäuser, Boston, 1990, 85-124. Zbl 0739.22012 MR 1103588 
[43] M. Moriwaki, Multiplicity-free decompositions of the minimal representation of the indefinite orthogonal group Int. J. Math. 19 (2008), 1187-1201. Zbl 1171.22012 MR 2466561

[44] G. I. Ol'shanskiı̌, Complex Lie semigroups, Hardy spaces and the Gel'fand-Gindikin program, Differential Geom. Appl. 1 (1991), 235-246. Zbl 0789.22011 MR 1244445

[45] M. Rösler, An uncertainty principle for the Dunkl transform, Bull. Austral. Math. Soc. 59 (1999), 353-360. Zbl 0939.33012 MR 1698045

[46] H. Sabourin, Une représentation unipotente associée à l'orbite minimale: le cas de $S O(4,3)$, J. Funct. Anal. 137 (1996), 394-465. Zbl 0849.22016 MR 1387517

[47] M. Sato, Theory of hyperfunctions. I, J. Fac. Sci. Univ. Tokyo Sect. I 8 (1959), 139-193. Zbl 0087.31402 MR 0114124

[48] R. J. Stanton, Analytic extension of the holomorphic discrete series, Amer. J. Math. 108 (1986), 1411-1424. Zbl 0626.43008 MR 0868896

[49] I. T. Todorov, Derivation and solution of an infinite-component wave equation for the relativistic Coulomb problem, in Group representations in mathematics and physics (Seattle, 1969), Lecture Notes in Phys. 6, Springer, 1970, 254-278. Zbl 0207.27604 MR 0339705

[50] P. Torasso, Méthode des orbites de Kirillov-Duflo et représentations minimales des groupes simples sur un corps local de caractéristique nulle, Duke Math. J. 90 (1997), 261-377. Zbl 0941.22017|MR 1484858

[51] D. A. Vogan, Jr., Singular unitary representations, in Noncommutative harmonic analysis and Lie groups (Marseille, 1980), Lecture Notes in Math. 880, Springer, Berlin, 1981, 506-535. Zbl 0464.22007 MR 0644845

[52] C.-B. Zhu and J.-S. Huang, On certain small representations of indefinite orthogonal groups, Represent. Theory 1 (1997), 190-206. Zbl 0887.22016 MR 1457244 